Article

\title{
Diet Influences the Oral Microbiota of Infants during the First Six Months of Life
}

\author{
Patrícia M. Oba ${ }^{1}{ }^{(\mathbb{D}, \text { Hannah D. Holscher }}{ }^{2,3}{ }^{\mathbb{D}}$, Rose Ann Mathai ${ }^{4}$, Juhee Kim ${ }^{5}$ \\ and Kelly S. Swanson $1,3, *$ (D) \\ 1 Department of Animal Sciences, University of Illinois at Urbana-Champaign, Urbana, IL 61801, USA; \\ obapm@illinois.edu \\ 2 Department of Food Science and Human Nutrition, University of Illinois at Urbana-Champaign, \\ Urbana, IL 61801, USA; hholsche@illinois.edu \\ 3 Division of Nutritional Sciences, University of Illinois at Urbana-Champaign, Urbana, IL 61801, USA \\ 4 Department of Nutrition, Dominican University, River Forest, IL 60305, USA; rmathai@dom.edu \\ 5 Department of Nutrition, East Carolina State University, Greenville, NC 27834, USA; kim.juhee@gmail.com \\ * Correspondence: ksswanso@illinois.edu; Tel.: +1-(217)-333-4189
}

Received: 29 September 2020; Accepted: 31 October 2020; Published: 5 November 2020

\begin{abstract}
Background: Oral microorganisms contribute to oral health and disease, but few have studied how infant feeding methods affect their establishment. Methods: Infant $(n=12)$ feeding records and tongue and cheek swabs were collected within $48 \mathrm{~h}$ of birth, and after 2, 4, and 6 mo. DNA was extracted from samples, bacterial and fungal amplicons were generated and sequenced using Illumina MiSeq, and sequences were analyzed using Quantitative Insights Into Microbial Ecology (QIIME) and Statistical Analysis System (SAS) to evaluate differences over time and among breast-fed, formula-fed, mixed-fed, and solid food-fed infants. Results: Considering all time points, breast milkand mixed-fed infants had lower oral species richness than solid food-fed infants $(p=0.006)$. Regardless of feeding mode, species richness was lower at birth than at other time points $(p=0.006)$. Principal coordinates analysis (PCoA) of unique fraction metric (UniFrac) distances indicated that bacterial communities were impacted by feeding method $(p<0.005)$. Considering all time points, breast-fed infants had higher Streptococcus, while formula-fed infants had higher Actinomyces and Prevotella. Regardless of feeding mode, Propionibacterium, Porphyromonas, Prevotella, Gemella, Granulicatella, Veillonella, Fusobacterium, Leptotrichia, Neisseria, and Haemophilus increased with age, while Cloacibacterium and Dechloromonas decreased with age. Oral fungi were detected in infants but were not impacted by diet. Conclusions: These findings demonstrate that the establishment of oral bacteria depends on dietary composition and age. More research is necessary to determine whether this affects risk of oral caries and other health outcomes later in life.
\end{abstract}

Keywords: oral bacteria and fungi; infant health; feeding method

\section{Introduction}

Breast milk is the first food that most newborn humans come in contact with. Human milk not only contains nutrients, but is rich in microbes and oligosaccharides that contribute to the early colonization of the gastrointestinal tract [1]. The breast milk microbiome can vary throughout lactation [2], but Streptococcus, Staphylococcus, Serratia, Pseudomonas, Corynebacteria, Ralstonia, Propionibacterium, Sphingomonas, and Bradyrhizobiaceae are the predominant genera present [3]. Infant saliva and breast milk of mother-child pairs both have a high abundance of Streptococcus spp. [4], with breast milk serving as an important source of microorganisms for the infant. However, around $5 \mathrm{mo}$ of age, an infant's oral microbiota differs from maternal microbiota and consists mostly of Streptococcus, Haemophilus, Neisseria, and Veillonella [5]. 
Clinical evidence suggests that the microbial colonization may begin in utero [6-9] and that delivery method influences the original seeding of microorganisms [10,11]. Therefore, the delivery mode affects the gastrointestinal tract microbiota of the infant [12-14], with vaginally delivered infants, at 3 mo of age, having an oral microbiota with higher taxonomic diversity than cesarean-born children [12]. Feeding method also affects the oral microbiota. There appear to be differences between breast-fed and formula-fed infants, with possible reasons for this being that breast-fed infants have higher lactobacilli populations that are capable of inhibiting the growth of Streptococcus [15]. Milk microbiota at 3-4 mo postpartum is dominated by Proteobacteria and Firmicutes, and milk microbiota composition and diversity are associated with maternal factors (body mass index, parity, and mode of delivery), breast feeding practices, and other milk components [16].

Information about fungal acquisition and maturation of the oral mycobiome is limited. The Candida genus is the most prevalent yeast in the oral cavity, with others in high abundance including Cladosporium, Aureobasidium, Saccharomycetales, Fusarium, Cryptococcus, and Aspergillus [17-19]. Candida spp. can be acquired by the infant through vertical transmission from the mother's vagina during birth $[14,20]$. Furthermore, it was reported that the increase of oral Candida load is associated with a low bacterial diversity, a dominance of Bacilli (streptococci and lactobacilli) and disappearance of Fusobacteria and Bacteroidia [21].

Oral bacteria and fungi contribute to oral health and establishment of the distal gastrointestinal tract microbiota [22-25]. However, there is a dearth of knowledge on the longitudinal changes that occur during the first few mo of life and how feeding method affects oral bacteria and fungi in infants. Therefore, we aimed to characterize the relationships between feeding method and oral bacterial and fungal communities in vaginally delivered infants during the first 6 mo of life. We hypothesized that the alpha diversity of the microbiota would increase over time, most notably with the addition of formula and/or solid foods. We hypothesized that (1) the infant oral bacterial and fungal microbiome would have a greater diversity with time, (2) the oral bacterial and fungal microbiome of each infant would have a greater diversity with the change from formula feeding to solid foods, and (3) similar to previous studies, Streptococcus would be the predominant oral bacterial genera in infants at all time points.

\section{Materials and Methods}

\subsection{Study Design}

This was a longitudinal, observational trial. Study procedures were administered in accordance with the Declaration of Helsinki and were approved by the University of Illinois at Urbana-Champaign Institutional Review Board (IRB \#11621; approved on 14 September 2011) and the Carle Foundation Hospital Institutional Review Board (IRB \#11020; approved on 7 December 2011) prior to participant recruitment. Healthy pregnant women who intended to breast feed their infants were recruited during their third semester of pregnancy (wk 26-40) in collaboration with the Carle Hospital Midwives. Healthy pregnant women were defined as women with uncomplicated pregnancy, such as those without preeclampsia, gestational diabetes, anemia, and no history of smoking. Infants were included in the study if they were vaginally delivered at $37 \mathrm{wk}$ of gestation or later. Study exclusion criteria included: cesarean section delivery, smoking during pregnancy, or any serious medical condition (e.g., preeclampsia, eclampsia).

\subsection{Study Participants and Sample Collection}

Samples from tongue and cheek were collected using a Catch-All Sample Collection Swab buccal swab (Epicentre Biotechnologies, Madison, WI), from a cohort of infants $(n=12)$ at four time points: at birth (within $48 \mathrm{~h}$ after delivery) and at $2 \mathrm{mo}, 4 \mathrm{mo}$, and $6 \mathrm{mo}$ of age. Two tongue samples were collected by brushing the center of the dorsum for $5 \mathrm{~s}$ and then placed into a sterile tube. Using separate swabs, two cheek samples were collected using a similar method. All samples were placed on ice, immediately transported to the laboratory, and then stored at $-80{ }^{\circ} \mathrm{C}$ until analyses. 
Feeding practices were separated as exclusive human milk, formula, mixed feeding (human milk + formula), and introduction to solid foods (mixed of solid foods + human milk/formula or both).

\subsection{DNA Extraction and Sequencing}

Sample DNA was extracted from all samples individually using the MoBio PowerSoil PowerLyzer kits (Mo Bio Laboratories, Inc., Carlsbad, CA, USA) according to manufacturer guidelines with minor modifications conducted in consultation with the manufacturer. Briefly, to increase DNA yield, columns were washed with $125 \mu \mathrm{L}$ of solution $\mathrm{C} 2$ and $125 \mu \mathrm{L}$ of solution $\mathrm{C} 3$ at the same time, rather than two steps, and a low elution spin filter (MoBio) was utilized to elute the DNA. After extraction, DNA quality was assessed using an E-Gel system (Invitrogen, Carlsbad, CA, USA) followed by quantification using a Qubit 2.0 Fluorometer (Life Technologies, Grand Island, NY, USA). Amplification of the variable region 3-5 (V3-V5) of the bacterial 16S rRNA (ribosomal ribonucleic acid) gene [26] and fungal internal transcribed spacer (ITS) 1-4 region [27] as conducted on a Fluidigm Access Array (Fluidigm Corporation, South San Francisco, CA, USA); CS1 forward tag and CS2 reverse tag were added according to the Fluidigm protocol. Quality of the amplicons was assessed using a Fragment Analyzer (Advanced Analytics, Ames, IA, USA) to confirm amplicon regions and sizes. A DNA pool was generated by combining equimolar amounts of the amplicons from each sample. The pooled samples were then size selected on a $2 \%$ agarose E-gel (Life technologies) and extracted using a Qiagen gel purification kit (Qiagen, Valencia, CA, USA). Cleaned, size-selected, pooled products were run on an Agilent Bioanalyzer (Advanced Analytics, Ames, IA, USA) to confirm appropriate profile and average size. Illumina sequencing was performed on a MiSeq using v3 reagents (Illumina Inc., San Diego, CA, USA) at the W. M. Keck Center for Biotechnology at the University of Illinois.

\subsection{Data Analyses}

Forward reads were trimmed using the FASTX-Toolkit (version 0.0.13), and Quantitative Insights Into Microbial Ecology (QIIME) 1.8 [28] was used to process the resulting sequence data. Briefly, high-quality (quality value $\geq 20$ ) sequence data derived from the sequencing process were demultiplexed. Sequences then were clustered into operational taxonomic units (OTU) using UCLUST [29] through a closed-reference OTU picking strategy against the Greengenes 13_8 reference database [30] with a 97\% similarity threshold. Singletons (OTU that were observed fewer than two times) and OTU that had less than $0.01 \%$ of the total observation were discarded. Taxonomic identity to each OTU was then assigned using UCLUST. An even sampling depth (sequences per sample) of 30,882 sequences per sample was used for assessing alpha diversity [observed species; phylogenetic diversity (PD_whole_tree); Chao1] and beta diversity measures. Beta diversity was calculated using weighted and unweighted unique fraction metric (UniFrac) [31] distance measures. Fungal sequences were picked using the pick_open_reference_otus.py command and the ITS_12_11_otus reference taxonomy provided by the UNITE database (https://unite.ut.ee).

All samples were collected, stored, and extracted separately. The samples were combined at the data analysis step because initial analysis showed that there were no statistical differences between the two points of collection. All data were analyzed using Statistical Analysis System (SAS, version 9.4, SAS Institute, Cary, NC, USA) using the Mixed Models procedure with dietary treatment and period being the fixed effect and infant being the random effect. Due to lack of samples for all feeding groups at all times, a time*diet interaction was not tested. When analyzing the main effect of diet, infants were separated into two groups: exclusive breast feeding until $4 \mathrm{mo}(n=6)$ and non-exclusive breast feeding until 4 mo $(n=6)$. Data normality was checked using the univariate procedure and Shapiro-Wilk statistic, with log transformation being used when normal distribution was lacking. If the data did not reach normality after a logarithmic transformation, the data were analyzed using the npar1way procedure and Wilcoxon statistic. Data were reported as means with $p<0.05$ being considered significant. As this was a preliminary trial, there were no corrections for multiple testing [32]. 


\section{Results}

A total of 10,899,630 16S rRNA-based amplicon sequences were obtained, with an average of 113,538 reads (range $=31,336-175,809$ ) per sample, rarefied at 30,882. On Table S1, there is the dietary information of infants at each time point and on Table S2, additional information of infants/mothers and delivery complications. Due to the lack of statistical differences between sample types, cheek and tongue sequence data were combined.

\subsection{Effect of Time on Infant Oral Microbiota}

Time had a significant influence on the oral bacterial phyla (Table 1), with Firmicutes being the phyla of highest prevalence in the infant oral cavity, accounting for $78.8 \pm 26.3 \%$ of all sequences. Firmicutes were higher $(p=0.0002)$ at 2 mo than other time points, followed by 4 mo, 6 mo, and birth that was the lowest. Actinobacteria were higher $(p=0.005)$ at birth compared to 4 mo and 6 mo. Bacteroidetes was higher $(p<0.0001)$ at 6 mo compared to birth and 2 mo, with 4 mo having an intermediate value. Fusobacteria were higher $(p<0.0001)$ at 6 mo than birth, 2 mo, and 4 mo, with 2 mo and 4 mo being greater than birth when these taxa were virtually absent. Proteobacteria were very high at birth, being higher $(p<0.0001)$ than $2 \mathrm{mo}, 4 \mathrm{mo}$, and 6 mo. Proteobacteria were also higher $(p<0.0001)$ at 6 mo compared to 2 and 4 mo.

Table 1. Oral bacterial phyla over time in human infants (\% of sequences).

\begin{tabular}{ccccccc}
\hline & \multicolumn{3}{c}{ Period $(\boldsymbol{n}=\mathbf{1 2})$} & \multicolumn{2}{c}{ Statistics } \\
\cline { 2 - 7 } Phyla & Birth & $\mathbf{2 ~ m o}$ & $\mathbf{4} \mathbf{~ m o}$ & $\mathbf{6}$ mo & SEM & $\begin{array}{c}\text { Period } \\
p \text {-Values }\end{array}$ \\
\hline Actinobacteria & $2.1^{\mathrm{a}}$ & $1.7^{\mathrm{ab}}$ & $1.4^{\mathrm{b}}$ & $1.3^{\mathrm{b}}$ & 0.85 & 0.005 \\
Bacteroidetes & $3.1^{\mathrm{c}}$ & $3.7^{\mathrm{c}}$ & $7.3^{\mathrm{b}}$ & $14.5^{\mathrm{a}}$ & 2.12 & $<0.0001$ \\
Firmicutes & $59.2^{\mathrm{z}}$ & $92.5^{\mathrm{w}}$ & $87.6^{\mathrm{x}}$ & $76.1^{\mathrm{y}}$ & 5.24 & 0.0002 \\
Fusobacteria & $0.0^{\mathrm{c}}$ & $0.5^{\mathrm{b}}$ & $1.6^{\mathrm{b}}$ & $2.5^{\mathrm{a}}$ & 0.56 & $<0.0001$ \\
Proteobacteria & $35.7^{\mathrm{a}}$ & $1.5^{\mathrm{c}}$ & $2.1^{\mathrm{c}}$ & $5.6^{\mathrm{b}}$ & 4.27 & $<0.0001$ \\
\hline
\end{tabular}

${ }^{\mathrm{a}-\mathrm{c}}$ Means with different superscripts within a row differ by Tukey's test $(p<0.05)$. ${ }^{\mathrm{w}-\mathrm{z}}$ Means with different superscripts within a row differ by Wilcoxon's test $(p<0.05)$.

Streptococcus spp. were by far the genera of highest prevalence in the infant oral cavity, accounting for $64.1 \pm 27.2 \%$ of all sequences (Table 2 ). In general, older infants had a higher abundance of Actinomyces, Porphyromonas, and Prevotella of the Prevotellaceae and Paraprevotellaceae families, unclassified Weeksellaceae, Gemella, Granulicatella, unclassified Streptococcaceae, unclassified Lachnospiraceae, Veillonella, Fusobacterium, Leptotrichia, Neisseria, and Haemophilus. In contrast, Cloacibacterium, unclassified Gemellaceae, unclassified Comamonadaceae, and Dechloromonas were more abundant at birth and, in general, decreased with age. Streptococcus was the only genus that had a lower concentration at birth and $6 \mathrm{mo}$ and higher at 2 and $4 \mathrm{mo}$, with the opposite pattern observed with Propionibacterium and Actinobacillus, which had a higher percentage at birth and 6 mo and lower at 2 and 4 mo (Table 2). 
Table 2. Oral bacterial genera of infants over time (\% of sequences).

\begin{tabular}{|c|c|c|c|c|c|c|c|c|}
\hline \multirow[b]{2}{*}{ Phyla } & \multirow[b]{2}{*}{ Family } & \multirow[b]{2}{*}{ Genus } & \multicolumn{4}{|c|}{ Period $(n=12)$} & \multicolumn{2}{|c|}{ Statistics } \\
\hline & & & Birth & $2 \mathrm{mo}$ & $4 \mathrm{mo}$ & $6 \mathrm{mo}$ & SEM & $\begin{array}{c}\text { Period } \\
p \text {-Values }\end{array}$ \\
\hline \multirow{2}{*}{ Actinobacteria } & Actinomycetaceae & Actinomyces & $0.0^{c}$ & $1.4^{\mathrm{b}}$ & $1.3^{\mathrm{a}}$ & $1.2^{\mathrm{a}}$ & 0.41 & $<0.0001$ \\
\hline & Propionibacteriaceae & Propionibacterium & $2.1^{\mathrm{w}}$ & $0.0^{\mathrm{z}}$ & $0.0^{\mathrm{y}}$ & $0.0^{x}$ & 0.66 & 0.006 \\
\hline \multirow{5}{*}{ Bacteroidetes } & Porphyromonadaceae & Porphyromonas & $0.0^{\mathrm{c}}$ & $0.4^{\mathrm{b}}$ & $1.611^{\mathrm{b}}$ & $2.6^{\mathrm{a}}$ & 0.49 & $<0.0001$ \\
\hline & Prevotellaceae & Prevotella & $0.0^{\mathrm{c}}$ & $2.6^{\mathrm{b}}$ & $3.050^{\mathrm{a}}$ & $4.2^{\mathrm{a}}$ & 1.45 & $<0.0001$ \\
\hline & Paraprevotellaceae & Prevotella & $0.0^{\mathrm{c}}$ & $0.5^{\mathrm{bc}}$ & $2.030^{\mathrm{b}}$ & $5.6^{\mathrm{a}}$ & 1.40 & $<0.0001$ \\
\hline & \multirow{2}{*}{ Weeksellaceae } & Unclassified & $0.0^{\mathrm{b}}$ & $0.3^{\mathrm{a}}$ & $0.625^{\mathrm{a}}$ & $1.6^{\mathrm{a}}$ & 0.34 & $<0.0001$ \\
\hline & & Cloacibacterium & $3.0^{\mathrm{a}}$ & $0.0^{\mathrm{b}}$ & $0.0^{\mathrm{b}}$ & $0.0^{\mathrm{b}}$ & 0.63 & $<0.0001$ \\
\hline \multirow{9}{*}{ Firmicutes } & Staphylococcaceae & Staphylococcus & 1.8 & 0.7 & 0.5 & 0.6 & 0.34 & 0.460 \\
\hline & \multirow{2}{*}{ Gemellaceae } & Unclassified & $9.4^{\mathrm{a}}$ & $4.0^{\mathrm{a}}$ & $4.1^{\mathrm{a}}$ & $4.2^{\mathrm{b}}$ & 2.04 & 0.049 \\
\hline & & Gemella & $0.1^{\mathrm{z}}$ & $0.1^{\mathrm{y}}$ & $0.3^{x}$ & $0.7^{\mathrm{w}}$ & 0.16 & $<0.0001$ \\
\hline & Carnobacteriaceae & Granulicatella & $0.0^{\mathrm{d}}$ & $0.3^{c}$ & $1.6^{\mathrm{b}}$ & $4.0^{\mathrm{a}}$ & 0.42 & $<0.0001$ \\
\hline & Lactobacillaceae & Lactobacillus & 0.0 & 1.3 & 0.0 & 0.0 & 0.48 & 0.594 \\
\hline & \multirow{2}{*}{ Streptococcaceae } & Unclassified & $0.0^{c}$ & $0.0^{\mathrm{c}}$ & $0.2^{b}$ & $0.4^{\mathrm{a}}$ & 0.04 & $<0.0001$ \\
\hline & & Streptococcus & $47.2^{b}$ & $78.9^{a}$ & $74.2^{\mathrm{a}}$ & $56.1^{\mathrm{b}}$ & 5.36 & $<0.0001$ \\
\hline & Lachnospiraceae & Unclassified & $0.3^{z}$ & $0.9 \mathrm{y}$ & $0.6^{x}$ & $0.3^{\mathrm{w}}$ & 0.41 & 0.021 \\
\hline & Veillonellaceae & Veillonella & $0.0^{b}$ & $6.3^{\mathrm{a}}$ & $5.7^{\mathrm{a}}$ & $9.7^{\mathrm{a}}$ & 1.40 & $<0.0001$ \\
\hline \multirow{2}{*}{ Fusobacteria } & Fusobacteriaceae & Fusobacterium & $0.0^{c}$ & $0.3^{b c}$ & $0.4^{\mathrm{ab}}$ & $0.9^{\mathrm{a}}$ & 0.26 & $<0.0001$ \\
\hline & Leptotrichiaceae & Leptotrichia & $0.0^{\mathrm{c}}$ & $0.1^{\mathrm{c}}$ & $1.2^{b}$ & $1.6^{\mathrm{a}}$ & 0.52 & $<0.0001$ \\
\hline \multirow{5}{*}{ Proteobacteria } & Comamonadaceae & Unclassified & $8.5^{a}$ & $0.0^{\mathrm{b}}$ & $0.0^{\mathrm{b}}$ & $0.0^{\mathrm{b}}$ & 1.79 & $<0.0001$ \\
\hline & Neisseriaceae & Neisseria & $3.3^{b}$ & $0.7^{\mathrm{b}}$ & $0.3^{b}$ & $3.4^{\mathrm{a}}$ & 0.99 & $<0.0001$ \\
\hline & Rhodocyclaceae & Dechloromonas & $1.6^{\mathrm{a}}$ & $0.0^{b}$ & $0.0^{\mathrm{b}}$ & $0.0^{\mathrm{b}}$ & 0.33 & $<0.0001$ \\
\hline & \multirow{2}{*}{ Pasteurellaceae } & Actinobacillus & $11.4^{\mathrm{w}}$ & $0.0^{\mathrm{y}}$ & $0.0^{z}$ & $0.0^{x}$ & 3.04 & 0.002 \\
\hline & & Haemophilus & $8.0^{\mathrm{z}}$ & $0.5^{\mathrm{y}}$ & $1.5^{x}$ & $1.9^{\mathrm{w}}$ & 2.32 & 0.017 \\
\hline
\end{tabular}

${ }^{\mathrm{a}-\mathrm{d}}$ Means with different superscripts within a row differ by Tukey's test $(p<0.05)$. ${ }^{\mathrm{w}-\mathrm{z}}$ Means with different superscripts within a row differ by Wilcoxon's test $(p<0.05)$. 
Species richness differed between birth and 2 mo $(p=0.006)$, birth and 4 mo $(p=0.006)$, birth and $6 \mathrm{mo}(p=0.006), 2 \mathrm{mo}$ and $6 \mathrm{mo}(p=0.006)$, and $4 \mathrm{mo}$ and $6 \mathrm{mo}(p=0.018$; Figure $1 \mathrm{~A})$, but did not differ between the 2 mo and $4 \mathrm{mo}(p=0.144)$. Unweighted and weighted principal coordinates analysis (PCoA) for infant age tended to cluster birth and 6 mo separately (Figure 1B,C).

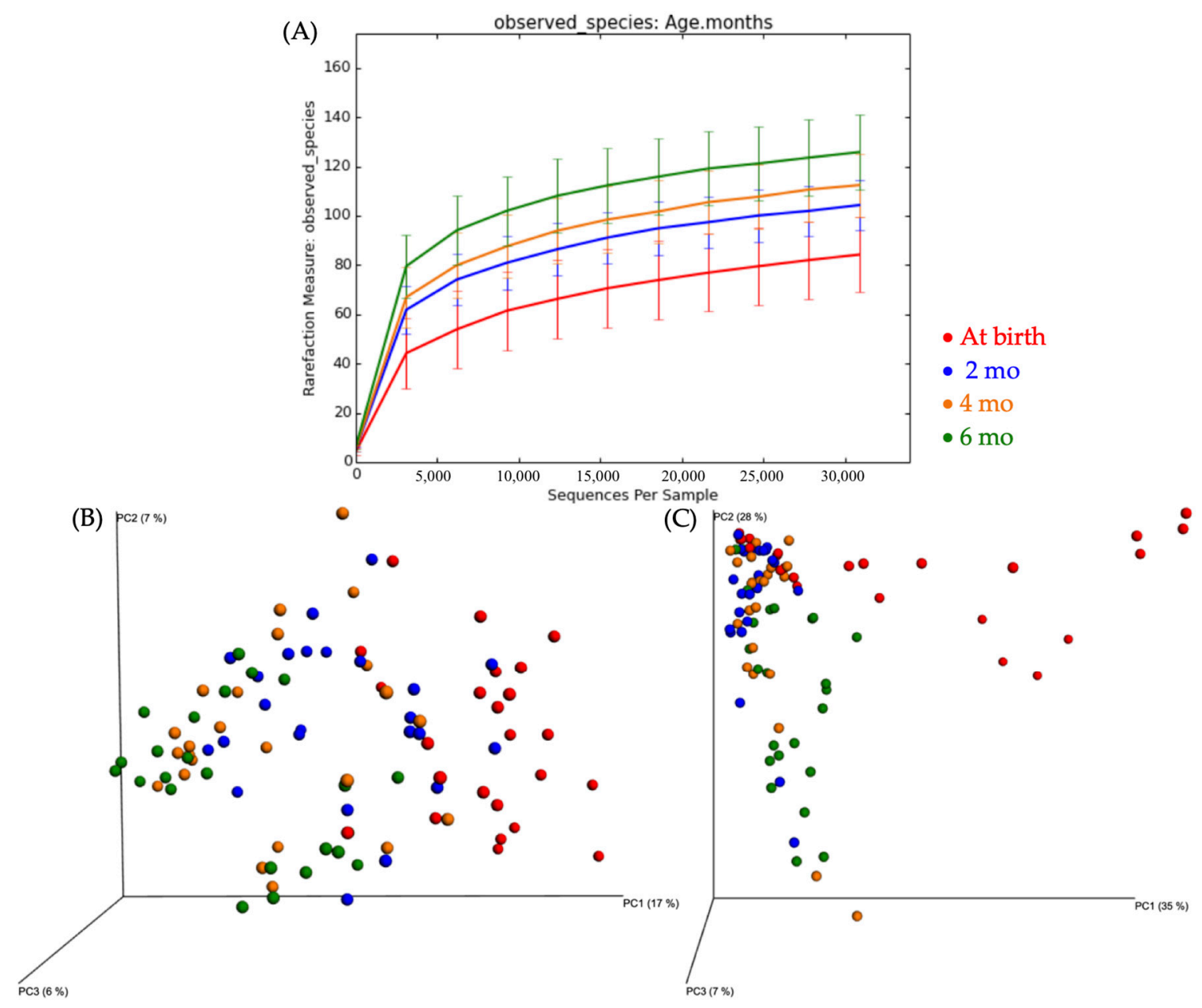

Figure 1. (A) Rarefaction curves showing bacterial species richness based on infant age. Mean number of operational taxonomic units (OTU) per subject for infant age. (B) Unweighted principal coordinates analysis (PCoA) plot of identified bacterial OTUs, shows plot for infant age. (C) Weighted PCoA plot of identified bacterial OTUs, shows plot for infant age.

Oral fungal communities were impacted by infant age (Table 3). Five infants had detectable levels of fungi within 24-48 h of birth, which changed to four, nine, and 10 at 2, 4, and 6 mo of age, respectively. Ascomycota counts were higher $(p=0.0475)$ at 2 mo of age than 4 and 6 mo of age, with infants at birth having intermediate values. Ascomycota was present in infants even within the first $24-48 \mathrm{~h}$ after birth (detected in $41.67 \%$ of infants in at least one site of collection), including $33 \%$ of infants by the age of $2 \mathrm{mo}, 67 \%$ of infants by the age of $4 \mathrm{mo}$, and $83 \%$ of infants by the age of $6 \mathrm{mo}$. Basidiomycota was detected in $33 \%$ of infants at birth, $17 \%$ of infants at $2 \mathrm{mo}, 33 \%$ of infants at $4 \mathrm{mo}$, and $58 \%$ of infants at 6 mo. Candida spp. were detected at a low frequency at birth (detected in $17 \%$ of infants in at least one site of collection), in $25 \%$ of infants at 2 and $4 \mathrm{mo}$, and $75 \%$ of infants by the age of 6 mo.

\subsection{Effect of Diet on Infant Oral Microbiota}

Length of breast feeding and exclusive breast feeding until for 4 mo did not have a significant influence on the bacterial phyla populations at any age (Table 4). When comparing the effect of receiving 
breast milk as an exclusive source of food within the first 24-48 h after birth, our results revealed that infants exclusively breast-fed had lower Haemophilus concentrations than those receiving a mix of formula and milk (Table S3). Overall, exclusively breast-fed infants had higher Neisseria $(p=0.01)$ and Actinobacillus $(p=0.0001)$ at birth, Streptococcus $(p=0.02)$ by the age of 2 mo, and unclassified Weeksellaceae $(p=0.0001)$ abundance by the age of 6 mo when compared to infants receiving mixed feedings. However, infants receiving only breast milk had lower Veillonella $(p=0.01)$ abundance by the age of 2 mo than those receiving mixed feedings (Table 5). Infants who received solid foods had higher abundance of Gemella ( $p=0.0003)$, unclassified Streptococcaceae $(p<0.0001)$, Veillonella $(p<0.0001)$, Fusobacterium $(p=0.006)$, and Neisseria $(p=0.0004)$. Streptococcus $(p=0.02)$, Actinobacillus $(p=0.003)$, and unclassified Gemellaceae $(p=0.02)$ were higher for breast-fed infants and lower for solid food-fed, and Actinomyces ( $p=0.0002)$, Prevotella $(p<0.0001)$, and unclassified Lachnospiraceae $(p<0.0001)$ were higher for formula-fed infants (Table 6).

Table 3. Infant oral fungi categorized over time (\% of sequences).

\begin{tabular}{ccccccc}
\hline \multirow{2}{*}{ Phylum } & \multicolumn{3}{c}{ Period } & \multicolumn{2}{c}{ Statistics } \\
\cline { 2 - 7 } & Birth & $\mathbf{2}$ mo & $\mathbf{4}$ mo & $\mathbf{6}$ mo & SEM & $\begin{array}{c}\text { Period } \\
p \text {-Values }\end{array}$ \\
\hline Ascomycota & $42^{\mathrm{ab}}$ & $83^{\mathrm{a}}$ & $22^{\mathrm{b}}$ & $26^{\mathrm{b}}$ & 14.5 & 0.047 \\
Basidiomycota & 28 & 19.68 & 21 & 16 & 12.0 & 0.755 \\
Unidentified & 5 & 1 & 28 & 26 & 11.1 & 0.194 \\
\hline
\end{tabular}

${ }^{\mathrm{a}, \mathrm{b}}$ Means with different superscripts within a row differ by Tukey's test $(p<0.05)$.

Table 4. Oral bacterial phyla of infants by age and duration of breast feeding (\% of sequences).

\begin{tabular}{|c|c|c|c|c|c|c|c|c|c|c|}
\hline \multirow{3}{*}{ Phyla } & \multicolumn{4}{|c|}{$\begin{array}{l}\text { Exclusive Breast Feeding for at Least } \\
\qquad 4 \operatorname{mo}(n=6)\end{array}$} & \multicolumn{4}{|c|}{ Breast-Fed for Less than 4 mo $(n=6)$} & \multicolumn{2}{|c|}{ Statistics } \\
\hline & \multicolumn{8}{|c|}{ Period } & \multirow{2}{*}{ SEM } & \multirow{2}{*}{$\begin{array}{c}\text { Diet } \times \text { Period } \\
p \text {-Values }\end{array}$} \\
\hline & Birth & $2 \mathrm{mo}$ & $4 \mathrm{mo}$ & $6 \mathrm{mo}$ & Birth & $2 \mathrm{mo}$ & $4 \mathrm{mo}$ & $6 \mathrm{mo}$ & & \\
\hline Actinobacteria & 0.1 & 0.6 & 1.3 & 1.5 & 4.1 & 2.9 & 1.6 & 1.0 & 1.17 & 0.148 \\
\hline Bacteroidetes & 3.4 & 1.1 & 1.9 & 11.3 & 2.8 & 6.4 & 12.8 & 17.7 & 2.80 & 0.194 \\
\hline Firmicutes & 54.8 & 95.1 & 93.6 & 78.5 & 63.5 & 89.8 & 81.6 & 73.7 & 7.55 & 0.342 \\
\hline Fusobacteria & 0.0 & 0.7 & 0.1 & 2.3 & 0.0 & 0.3 & 3.0 & 2.7 & 0.78 & 0.135 \\
\hline Proteobacteria & 41.7 & 2.5 & 3.1 & 6.4 & 29.7 & 0.6 & 1.0 & 4.9 & 6.13 & 0.641 \\
\hline
\end{tabular}


Table 5. Oral bacterial genera of infants by age and duration of breast feeding ( $\%$ of sequences).

\begin{tabular}{|c|c|c|c|c|c|c|c|c|c|c|c|c|}
\hline \multirow[b]{2}{*}{ Phyla } & \multirow[b]{2}{*}{ Family } & \multirow[b]{2}{*}{ Genus } & \multicolumn{4}{|c|}{ Breast-Fed at Least $4 \mathrm{mo}(n=6)$} & \multicolumn{4}{|c|}{ Breast-Fed for Less than $4 \mathrm{mo}(n=6)$} & \multicolumn{2}{|c|}{ Statistics } \\
\hline & & & Birth & $2 \mathrm{mo}$ & $4 \mathrm{mo}$ & $6 \mathrm{mo}$ & Birth & $2 \mathrm{mo}$ & $4 \mathrm{mo}$ & $6 \mathrm{mo}$ & SEM & $\begin{array}{c}\text { Diet } \times \text { Period } \\
p \text {-Values }\end{array}$ \\
\hline \multirow{2}{*}{ Actinobacteria } & Actinomycetaceae & Actinomyces & 0.0 & 0.5 & 1.2 & 1.4 & 0.0 & 2.2 & 1.4 & 1.0 & 0.58 & 0.051 \\
\hline & Propionibacteriaceae & Propionibacterium & $0.1^{\mathrm{ab}}$ & $0.0^{\mathrm{b}}$ & $0.0^{\mathrm{b}}$ & $0.0^{\mathrm{b}}$ & $4.0^{\mathrm{a}}$ & $0.0^{\mathrm{b}}$ & $0.0^{\mathrm{b}}$ & $0.0^{\mathrm{b}}$ & 0.90 & 0.003 \\
\hline \multirow{5}{*}{ Bacteroidetes } & Porphyromonadaceae & Porphyromonas & 0.0 & 0.6 & 0.4 & 3.2 & 0.0 & 0.1 & 2.8 & 2.1 & 0.69 & 0.129 \\
\hline & Prevotellaceae & Prevotella & 0.0 & 0.1 & 0.2 & 0.6 & 0.0 & 5.1 & 5.9 & 7.9 & 1.82 & 0.051 \\
\hline & Paraprevotellaceae & Prevotella & 0.0 & 0.0 & 0.1 & 4.5 & 0.0 & 0.9 & 3.9 & 6.8 & 2.01 & 0.436 \\
\hline & \multirow{2}{*}{ Weeksellaceae } & Unclassified & $0.0^{\mathrm{d}}$ & $0.3^{a b c}$ & $1.1^{\mathrm{ab}}$ & $3.0^{\mathrm{a}}$ & $0.1^{\mathrm{cd}}$ & $0.2^{\mathrm{c}}$ & $0.2^{\mathrm{c}}$ & $0.2^{b}$ & 0.41 & 0.0001 \\
\hline & & Cloacibacterium & $3.2^{\mathrm{ab}}$ & $0.0^{\mathrm{bc}}$ & $0.0^{\mathrm{bc}}$ & $0.1^{b c}$ & $2.7^{\mathrm{a}}$ & $0.0^{\mathrm{b}}$ & $0.0^{\mathrm{c}}$ & $0.0^{\mathrm{c}}$ & 0.92 & 0.009 \\
\hline \multirow{9}{*}{ Firmicutes } & Staphylococcaceae & Staphylococcus & 0.8 & 1.0 & 0.6 & 0.5 & 2.8 & 0.4 & 0.5 & 0.6 & 0.47 & 0.829 \\
\hline & \multirow{2}{*}{ Gemellaceae } & Unclassified & 14.0 & 3.6 & 4.6 & 3.7 & 4.7 & 4.4 & 3.5 & 4.6 & 2.87 & 0.348 \\
\hline & & Gemella & 0.0 & 0.0 & 0.5 & 1.2 & 0.2 & 0.1 & 0.1 & 0.3 & 0.22 & 0.082 \\
\hline & Carnobacteriaceae & Granulicatella & 0.0 & 0.1 & 0.5 & 4.3 & 0.0 & 0.4 & 2.6 & 3.8 & 0.58 & 0.083 \\
\hline & Lactobacillaceae & Lactobacillus & 0.0 & 0.5 & 0.0 & 0.0 & 0.0 & 2.0 & 0.0 & 0.0 & 0.68 & 0.936 \\
\hline & \multirow{2}{*}{ Streptococcaceae } & Unclassified & 0.0 & 0.0 & 0.1 & 0.4 & 0.0 & 0.1 & 0.3 & 0.3 & 0.06 & 0.370 \\
\hline & & Streptococcus & $39.8^{c}$ & $87.5^{\mathrm{a}}$ & $84.7^{\mathrm{ab}}$ & $60.9^{b c}$ & $54.5^{b c}$ & $70.3^{b c}$ & $63.7^{b c}$ & $51.3^{\mathrm{c}}$ & 7.35 & 0.021 \\
\hline & Lachnospiraceae & Unclassified & 0.0 & 0.0 & 0.0 & 0.2 & 0.7 & 1.8 & 1.2 & 0.5 & 0.56 & 0.156 \\
\hline & Veillonellaceae & Veillonella & $0.0^{\mathrm{d}}$ & $2.3^{b c}$ & $2.5^{b}$ & $7.2^{\mathrm{ab}}$ & $0.0^{\mathrm{cd}}$ & $10.3^{\mathrm{a}}$ & $8.8^{\mathrm{ab}}$ & $12.1^{a}$ & 1.70 & 0.010 \\
\hline \multirow{2}{*}{ Fusobacteria } & Fusobacteriaceae & Fusobacterium & 0.0 & 0.7 & 0.1 & 1.2 & 0.0 & 0.0 & 0.7 & 0.6 & 0.37 & 0.194 \\
\hline & Leptotrichiaceae & Leptotrichia & $0.0^{\mathrm{d}}$ & $0.0^{\mathrm{d}}$ & $0.0^{\mathrm{cd}}$ & $1.1^{b c}$ & $0.0^{\mathrm{d}}$ & $0.2^{\mathrm{cd}}$ & $2.3^{b c}$ & $2.2^{\mathrm{ab}}$ & 0.72 & 0.025 \\
\hline \multirow{5}{*}{ Proteobacteria } & Comamonadaceae & Unclassified & $8.7^{\mathrm{a}}$ & $0.0^{\mathrm{b}}$ & $0.1^{\mathrm{b}}$ & $0.1^{\mathrm{b}}$ & $8.3^{a}$ & $0.0^{\mathrm{b}}$ & $0.0^{\mathrm{b}}$ & $0.0^{\mathrm{b}}$ & 2.61 & 0.001 \\
\hline & Neisseriaceae & Neisseria & $6.5^{\mathrm{ab}}$ & $1.3^{\mathrm{abc}}$ & $0.3^{c}$ & $4.4^{\mathrm{a}}$ & $0.0^{\mathrm{c}}$ & $0.2^{b}$ & $0.4^{\mathrm{c}}$ & $2.5^{\mathrm{a}}$ & 1.29 & 0.011 \\
\hline & Rhodocyclaceae & Dechloromonas & $1.3^{\mathrm{ab}}$ & $0.0^{b c}$ & $0.0^{\mathrm{bc}}$ & $0.0^{\mathrm{c}}$ & $1.8^{\mathrm{a}}$ & $0.0^{\mathrm{bc}}$ & $0.0^{b c}$ & $0.0^{\mathrm{c}}$ & 0.49 & 0.010 \\
\hline & \multirow{2}{*}{ Pasteurellaceae } & Actinobacillus & $22.7^{\mathrm{a}}$ & $0.0^{\mathrm{bc}}$ & $0.0^{\mathrm{b}}$ & $0.0^{\mathrm{b}}$ & $0.0^{\mathrm{b}}$ & $0.0^{\mathrm{b}}$ & $0.0^{\mathrm{b}}$ & $0.1^{\mathrm{b}}$ & 4.03 & 0.0001 \\
\hline & & Haemophilus & $0.0^{\mathrm{b}}$ & $0.7^{\mathrm{ab}}$ & $2.5^{\mathrm{ab}}$ & $1.9^{\mathrm{a}}$ & $15.9^{a b}$ & $0.3^{\mathrm{ab}}$ & $0.5^{\mathrm{ab}}$ & $2.0^{\mathrm{ab}}$ & 3.14 & 0.041 \\
\hline
\end{tabular}

-d Means with different superscripts within a row differ by Tukey's test $(p<0.05)$. 
Table 6. Oral bacterial genera of infants by feeding method (\% of sequences).

\begin{tabular}{|c|c|c|c|c|c|c|c|c|}
\hline \multirow[b]{2}{*}{ Phyla } & \multirow[b]{2}{*}{ Family } & \multirow[b]{2}{*}{ Genus } & \multicolumn{4}{|c|}{ Diet } & \multicolumn{2}{|c|}{ Statistics } \\
\hline & & & $\begin{array}{c}\text { Human } \\
\text { Milk }\end{array}$ & Formula & Mixed & Solids & SEM & $\begin{array}{c}\text { Diet } \\
p \text {-Values }\end{array}$ \\
\hline Actinobacteria & Actinomycetaceae & Actinomyces & $0.8^{z}$ & $1.5^{\mathrm{w}}$ & $0.8^{y}$ & $1.3^{x}$ & 0.44 & 0.0002 \\
\hline \multirow{2}{*}{ Bacteroidetes } & Prevotellaceae & Prevotella & $0.2^{\mathrm{z}}$ & $14.8^{\mathrm{w}}$ & $2.0 \mathrm{y}$ & $5.3^{x}$ & 1.26 & $<0.0001$ \\
\hline & Weeksellaceae & Unclassified & 0.0 & 0.9 & 0.7 & 1.7 & 0.40 & 0.105 \\
\hline \multirow{7}{*}{ Firmicutes } & Staphylococcaceae & Staphylococcus & 1.1 & 0.2 & 1.0 & 0.5 & 0.35 & 0.609 \\
\hline & \multirow{2}{*}{ Gemellaceae } & Unclassified & $5.8^{\mathrm{a}}$ & $2.9^{a b}$ & $6.6^{\mathrm{ab}}$ & $4.0^{\mathrm{b}}$ & 2.14 & 0.023 \\
\hline & & Gemella & $0.0^{\mathrm{y}}$ & $0.3^{\mathrm{z}}$ & $0.3^{x}$ & $0.7^{\mathrm{w}}$ & 0.18 & 0.0003 \\
\hline & \multirow{2}{*}{ Streptococcaceae } & Unclassified & $0.0^{\mathrm{b}}$ & $0.4^{\mathrm{ab}}$ & $0.2^{b}$ & $0.4^{\mathrm{a}}$ & 0.05 & $<0.0001$ \\
\hline & & Streptococcus & $68.4^{\mathrm{w}}$ & $67.3^{y}$ & $65.1^{x}$ & $54.4^{\mathrm{z}}$ & 5.99 & 0.021 \\
\hline & Lachnospiraceae & Unclassified & $0.2^{\mathrm{z}}$ & $5.5^{\mathrm{w}}$ & $0.5^{\mathrm{y}}$ & $0.4^{x}$ & 0.32 & $<0.0001$ \\
\hline & Veillonellaceae & Veillonella & $2.5^{\mathrm{z}}$ & $5.1^{\mathrm{y}}$ & $6.9^{x}$ & $9.7^{\mathrm{w}}$ & 1.40 & $<0.0001$ \\
\hline Fusobacteria & Fusobacteriaceae & Fusobacterium & $0.1^{b}$ & $0.5^{\mathrm{ab}}$ & $0.5^{\mathrm{ab}}$ & $0.8^{\mathrm{a}}$ & 0.29 & 0.006 \\
\hline \multirow{2}{*}{ Proteobacteria } & Neisseriaceae & Neisseria & $2.3^{x}$ & $0.1^{\mathrm{y}}$ & $0.1^{\mathrm{z}}$ & $3.3^{\mathrm{w}}$ & 0.95 & 0.0004 \\
\hline & Pasteurellaceae & Actinobacillus & $5.4^{x}$ & $0.2^{\mathrm{z}}$ & $0.5^{\mathrm{y}}$ & $0.7^{\mathrm{w}}$ & 3.11 & 0.003 \\
\hline
\end{tabular}

or both. ${ }^{a, b}$ Means with different superscripts within a row differ by Tukey's test $(p<0.05)$. ${ }^{\mathrm{w}-\mathrm{z}}$ Means with different superscripts within a row differ by Wilcoxon's test $(p<0.05)$. 
Considering all time points, the species richness differed between the solid food-vs. human milk-fed groups $(p=0.006)$ and solid food- vs. mixed-fed groups $(p=0.006$; Figure 2A). Species richness did not differ between the human milk- and mixed-fed groups, solid- and formula-fed groups, mixed- and formula-fed groups, or human milk- and formula-fed groups. Unweighted and weighted PCoA for feeding method tended to cluster the solid food feeding method separately, and with human milk being separate from mixed feeding method (Figure 2B; Figure 2C).

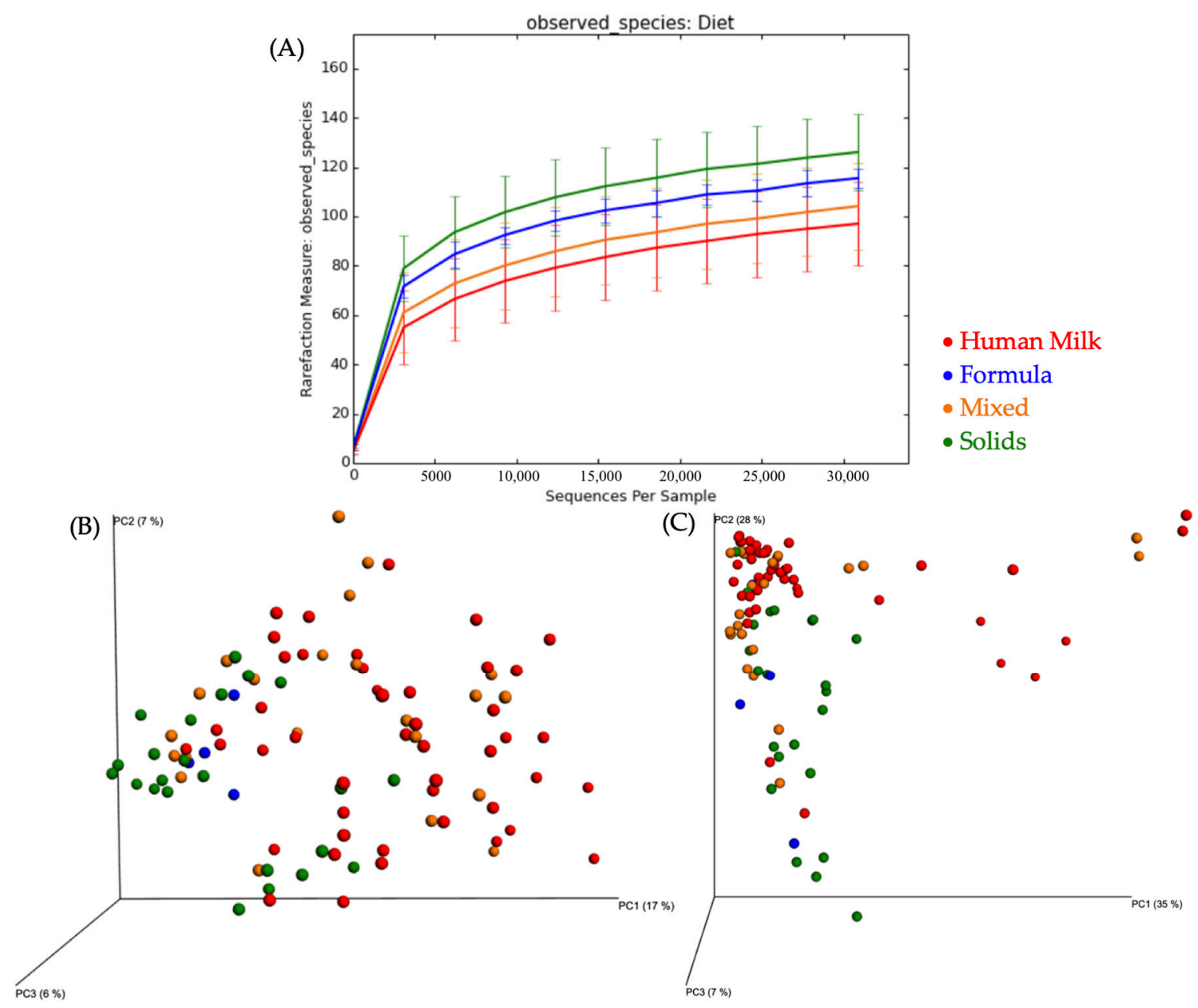

Figure 2. (A) Rarefaction curves showing bacterial species richness based on feeding method. Mean number of bacterial operational taxonomic units (OTU) per subject for feeding method.

(B) Unweighted PCoA plot of identified bacterial OTUs, shows plot for feeding method. (C) Weighted PCoA plot of identified bacterial OTUs, shows plot for feeding method.

Fungal communities of all samples are represented on Figure 3. Fungal communities were not impacted by diet (Table 7). Ascomycota and Basidiomycota were not detected in infants fed formula. Ascomycota was detected in 52\% of infants fed human milk (in at least one point and site of collection), $36 \%$ of infants fed mixed diet, and $83 \%$ of infants fed solid foods. Basidiomycota was detected in $39 \%$ of infants fed human milk ((HM) in at least one point and site of collection), $9 \%$ of infants fed mixed diet, and $58 \%$ of infants fed solid foods. Candida spp. were detected at a low frequency in infants fed human milk (detected in $22 \%$ of infants in at least one point and site of collection), in $27 \%$ of infants receiving mixed diet, and in a higher frequency of infants fed solids foods (detected in $75 \%$ of infants). 


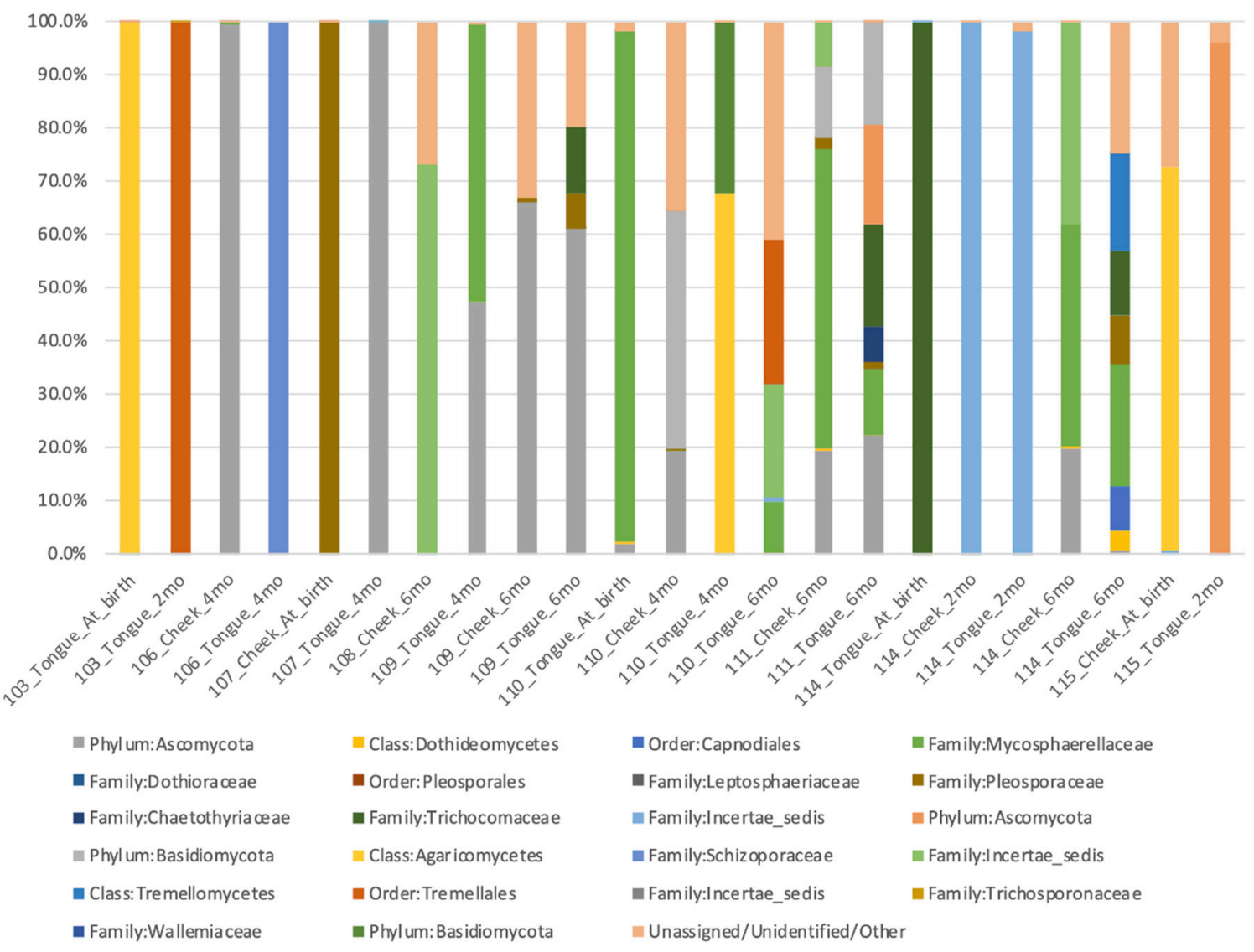

Figure 3. Fungal community ( $>5 \%$ classified) composition was diverse in infants.

Table 7. Infant oral fungal populations by feeding method ( $\%$ of sequences).

\begin{tabular}{cccccc}
\hline \multirow{2}{*}{ Phylum } & \multicolumn{3}{c}{ Diet } & \multicolumn{2}{c}{ Statistics } \\
\cline { 2 - 6 } & $\begin{array}{c}\text { Human } \\
\text { Milk }\end{array}$ & Mixed & Solids & SEM & $\begin{array}{c}\text { Diet } \\
\boldsymbol{p} \text {-Values }\end{array}$ \\
\hline Other & 4.9 & 3 & 11 & 7.4 & 0.20 \\
Ascomycota & 28 & 66 & 34 & 12.0 & 0.19 \\
Basidiomycota & 28 & 0 & 20 & 9.5 & 0.47 \\
Unidentified & 18 & 17 & 23 & 10.0 & 0.29
\end{tabular}

Human milk—exclusively fed with human milk, Mixed—fed with human milk + formula, Solids—fed with solid foods + human milk/formula or both.

\section{Discussion}

Studies using high-throughput sequencing methods to examine the oral microbiome of infants within 6 mo of age are rare $[5,11]$. To our knowledge, the current study is the only one using this technology to report relationships between feeding method and age on both bacterial and fungal communities in the infant oral cavity during this early stage of life.

As demonstrated in previous studies, the oral bacterial communities of infants were dominated by the phylum Firmicutes [5,33,34], being similar to results from children ( 3 to 6 years old) [35] and adults $[36,37]$ in previous studies. Proteobacteria were the second most abundant phyla, being similar to previous studies in adults $(>27 \%)$ [36] and infants $(>8 \%)$ [5]. The main difference between the current study and a previous study with infants (mean age $=4.6 \pm 1.2 \mathrm{mo}$ old) [5], children [35], and adults [37] was the higher prevalence of Bacteroidetes. In those populations, these phyla were present at an average relative abundance of $16-37 \%$. In the current study, it was only $7.16 \pm 9.77 \%$ of total sequences and more similar to that of Cephas et al. [5]. Cephas et al. [5] study used the V4-V6 region of $16 \mathrm{~S}$ 
rDNA genes, Ling et al. [35] used the V3 region of $16 \mathrm{~S}$ rRNA genes, and Keijser et al. [37] used the V6 region of 16S rRNA genes, and all were subjected to 454-pyrosequencing. In the present study, the V3-V5 regions of $16 \mathrm{~S}$ rRNA genes were used and subjected to Illumina sequencing, which may contribute to the reported differences.

At the genus level, our results are in agreement with previous studies of the oral microbiota of infants $\leq 6$ mo of age. Similar to the previous studies, the predominant genera present in samples of infants was Streptococcus spp. [5,33,34], which were detected in all infants from the ages of 2 to 24 mo [38]. The present study also detected Streptococcus spp. as the predominant genera and all infants and ages tested had the presence of this genus. In agreement with the current study, infants of 4-5 mo of age had a predominance of Streptococcus, Veillonella, Haemophilus, and Granulicatella being observed at average levels $>1 \%$ of sequences [5]. In contrast to the previous study in our laboratory, the infants of 4-5 mo of age did not have Propionibacterium, Porphyromonas, Prevotella, or Leptotrichia present at average levels $>1 \%$ of sequences [5]. Moreover, Neisseria, Rothia, Gemella, Leptotrichia, and Fusobacterium were not present in the current study, but were predominant genera in 4-mo-old infants in the previous study. Furthermore, Veillonella, Fusobacterium, Streptococcus, and Actinomyces, which are considered to be early colonizers of the oral cavity [39-41], were all detected in the oral cavity of infants tested in the present study in all periods (at birth, $2 \mathrm{mo}, 4 \mathrm{mo}$, and 6 mo of age). However, in birth, Actinomyces, Fusobacterium, and Veillonella have a low number of species in comparison with all the other periods.

Previous research has shown that the most abundant genera in human milk are Streptococcus and Staphylococcus [3,42]. Lactobacillus is also known to be a common member of the milk microbiota. In the present study, the most abundant genera in the oral cavity of infants fed human milk were Streptococcus, Actinobacillus, Neisseria, Veillonella, and Staphylococcus. Moreover, Lactobacillus was isolated from $100 \%$ of breast-fed infants (for at least one site of collection) at birth, $86 \%$ at $2 \mathrm{mo}, 83 \%$ at $4 \mathrm{mo}$, and in the only infant consuming human milk at 6 mo, however, at a low relative abundance. Breast milk most likely contributes to the population of Streptococcus, Staphylococcus, and Lactobacillus in the oral cavity of infants. Similar to previous studies, Prevotella spp. (phylum Bacteroidetes) were more abundant in the oral cavity of formula-fed infants when compared to breast-fed infants [33]. Additionally, it was reported that Lactobacillus spp. were not detected in formula-fed infants at the age of 3 mo [15]. Conversely, in the present study, Lactobacillus spp. were detectable in infants exclusively fed formula $(n=2)$ and in the majority of infants fed a mixture of formula and breast milk (4 of 5). Only two infants receiving a mixture of formula and breast milk lacked Lactobacillus spp. as part of their oral microbiota in one point of sampling (considering all points of samplings), but these genera were only present after 2 mo. when fed a mixture of formula and breast milk. One infant lacked oral Lactobacillus spp. when fed a mixture of formula and breast milk but had it when receiving exclusive breast milk or solid food. In a previous study, Bifidobacterium spp. were detected in the oral cavity of only one breast-fed ( 1 of 26) and two formula-fed (2 of 12) infants [33]. Similar in the current study, Bifidobacterium spp. were not measured in any of the mouths of breast-fed or formula-fed infants.

Children and young adults with dental caries have been reported to have higher concentrations of Veillonella, Lactobacillus, Bifidobacterium, Propionibacterium, low-pH non-S. mutans Streptococci, Actinomyces, and Atopobium. These bacteria are thought to impact the development and progression of dental caries [43]. Similarly, Actinomyces, Granulicatella, Veillonella, Bifidobacteriaceae, and Scardovia have been identified as contributors to early childhood caries [44-46]. In the present study, infants fed human milk exclusively for 4 mo had lower Veillonella concentrations by the age of 2 mo than those receiving mixed feedings. This may indicate that a longer period of exclusive breastfeeding would potentially benefit oral health in early life, as previous studies have reported that breastfeeding in infancy may protect against dental caries [47].

Another factor that can affect the oral microbiota of infants is birth delivery mode. Within seconds after delivery, Lactobacillus, Prevotella, Atopobium, and Sneathia spp. have been reported to be the most predominant oral bacteria in vaginally delivered infants [11]. In the present study, all infants were vaginally delivered. In the first samples, which were collected within $48 \mathrm{~h}$ after birth (Table S3), 
the predominant genera were Streptococcus, Actinobacillus, Haemophilus, Neisseria, Cloacibacterium, Propionibacterium, Staphylococcus, and Dechloromonas. Gram-positive cocci, including Streptococcus and Staphylococcus, have been reported to be some of the most frequent early colonizers of the oral cavity $[48,49]$. Our results suggest that even in a short period (within $48 \mathrm{~h}$ after delivery) the oral microbiota of infants changes drastically from Lactobacillus, Prevotella, Atopobium, and Sneathia spp. to those measured in our study, likely due to environmental exposures during the first few hours (e.g., provision of colostrum or formula, introduction of pacifiers, hospital conditions, contact with hospital staff and family, etc.). Another study reported that vaginally delivered infants at the age of 3 mo. had a higher proportion of Capnocytophaga (16\%), Prevotella (40\%), Leptotrichia (16\%), and Haemophilus $(28 \%)$ than caesarian section-delivered infants of the same age [12]. In the present study, 100\% of children had detectable levels of oral Haemophilus, Leptotrichia, and Prevotella at 2 mo of age. Only three (25\%) samples from the tongue and five (41.67\%) samples from the cheek, however, were positive for Capnocytophaga. These data suggest that environmental exposures early in life are more influential than delivery mode.

Fungi may also play a role in oral health. Candida spp. have been reported to be the most predominant yeast in the oral cavity, having a high frequency $(75 \%)$ in oral rinse samples collected from healthy adults (21-60 yr old), followed by Cladosporium (65\%), Aureobasidium (50\%), Saccharomycetales (50\%), Aspergillus (35\%), Fusarium (30\%), and Cryptococcus (20\%) [19]. All of those fungal genera, with the exception of Cryptococcus (phylum Basidiomycota), are part of the phylum Ascomycota. In another study comparing oral microbiota of healthy adults and adults with periodontal disease, it was reported that Candida and Aspergillus were the most frequently observed genera (isolated from 100\% of participants), followed by Penicillium (97\%), Schizophyllum (93\%), Rhodotorula (90\%), and Gibberella (83\%). Schizophyllum and Rhodotorula are members of the phylum Basidiomycota, while the others are members of Ascomycota. Peters et al. [50] reported that differences in overall oral mycobiome diversity or composition were not observed between participants with periodontal disease and participants with good oral health, but that Ascomycota had a higher frequency of sequence reads $(86.5 \%)$ in all oral wash samples. Human milk may contribute to the presence of fungi in the oral cavity. By comparing the human milk mycobiota extracted at two different points in time (7-15 days (transient milk) and 45-90 days (mature milk)), a recent study reported that the most abundant genera in transient milk were Aspergillus (44.2\%), Saccharomyces (35.8\%), and Malessezia (12.3\%), and in mature milk they were Aspergillus (57.9\%), Penicillium (18.6\%), and Malessezia (10.5\%) [51]. All of those fungal genera, with the exception of Malessezia (phylum Basidiomycota), are part of the phylum Ascomycota. To our knowledge, the current study is the first to evaluate the mycobiome diversity of infants from birth to 6 mo of life. Ascomycota and Basidiomycota were also measured in infants, even within the first 24-48 h after birth. In general, the frequency of yeast in the oral cavity increased with age. Additionally, Candida spp. were measured in a low frequency at birth, with a higher frequency of these yeasts as age increased. Furthermore, infants fed human milk or a mixture of human milk and formula had a lower frequency of Candida spp. than when infants were fed solid foods. These results may indicate that Ascomycota, Basidiomycota, and Candida spp. are regular members of the oral mycobiome, with effects coming from the type of diet consumed. More studies are needed to elucidate the role of yeast on oral health and the effects of food on the mycobiome.

This study evaluated bacteria and fungi in a longitudinal trial but did have a few limitations. Because a small number of infants were studied, and due to the experimental design and samples collected, this study cannot determine causality. Rather, it was only possible to identify associations among microbial taxa, infant age, and feeding strategy. In addition, no vaginal swabs, breast milk, and food offered (solid or formula) were collected. For this reason, it was not possible to correlate the contribution of the external microbial populations on the oral microbiota of infants. Finally, due to a lack of samples at each time point, testing diet $\times$ time interactions with sufficient power was not possible. 


\section{Conclusions}

In conclusion, the current study has provided novel information about the oral microbiota of healthy human infants from between birth and 6 mo of age and how microbiota changes relate to feeding method. Differences were detected in the taxonomic profiles of different feeding methods, with infants receiving solid foods having higher concentrations of Gemella, Veillonella, Fusobacterium, Neisseria, and Actinobacillus. Considering all time points, breast-fed infants had a higher concentration of Streptococcus, and formula-fed infants had a higher concentration of Actinomyces and Prevotella. We also noted alterations in the oral microbiota over time, with increased abundance of Propionibacterium, Porphyromonas, Prevotella, Gemella, Granulicatella, Veillonella, Fusobacterium, Leptotrichia, Neisseria, and Haemophilus with age, and a decrease of Cloacibacterium and Dechloromonas with age. In addition, the older infants had a higher frequency of oral yeasts, namely, Candida spp. In addition, breast-fed and mixed-fed infants had a lower frequency of Candida spp. than those fed solids foods. Our data suggest that environmental exposures early in life are more influential than delivery mode.

Supplementary Materials: The following are available online at http:/www.mdpi.com/2072-6643/12/11/3400/s1, Table S1: Dietary information of infants at each time point, Table S2: Additional information of infants/mothers, and delivery complications, Table S3. Influence of feeding method on bacterial genera at birth $(24-48 \mathrm{~h}$ after delivery).

Author Contributions: K.S.S., J.K., R.A.M., and H.D.H. contributed to the hypothesis development and study design; H.D.H. conducted the sample collection and analysis; P.M.O., K.S.S., and H.D.H. contributed to the manuscript preparation; P.M.O. and H.D.H. were responsible for the data analysis; P.M.O., H.D.H., and K.S.S. contributed to the data interpretation and manuscript revision. All authors have read and agreed to the published version of the manuscript.

Funding: This research was funded by United States Department of Agriculture (USDA) Hatch \#ILLU 538-937.

Acknowledgments: The authors would like to acknowledge all of the mothers and infants who contributed to this research.

Conflicts of Interest: The authors declare no conflict of interest.

\section{References}

1. Ballard, O.; Morrow, A.L. Human milk composition: Nutrients and bioactive factors. Pediatr. Clin. N. Am. 2013, 60, 49-74. [CrossRef] [PubMed]

2. Jeurink, P.V.; van Bergenhenegouwen, J.; Jiménez, E.; Knippels, L.M.J.; Fernández, L.; Garssen, J.; Knol, J.; Rodríguez, J.; Martín, R. Human milk: A source of more life than we imagine. Benef. Microbes 2013, 4, 17-30. [CrossRef]

3. Hunt, K.M.; Foster, J.A.; Forney, L.J.; Schütte, U.M.E.; Beck, D.L.; Abdo, Z.; Fox, L.K.; Williams, J.E.; Mcguire, M.K.; Mcguire, M.A. Characterization of the diversity and temporal stability of bacterial communities in human milk. PLoS ONE 2011, 6, e21313. [CrossRef] [PubMed]

4. Davé, V.; Street, K.; Francis, S.; Bradman, A.; Riley, L.; Eskenazi, B.; Holland, N. Bacterial microbiome of breast milk and child saliva from low-income Mexican-American women and children. Pediatr. Res. 2016, 79, 846-854. [CrossRef]

5. Cephas, K.D.; Kim, J.; Mathai, R.A.; Barry, K.A.; Dowd, S.E.; Meline, B.S.; Swanson, K.S. Comparative analysis of salivary bacterial microbiome diversity in edentulous infants and their mothers or primary care givers using pyrosequencing. PLoS ONE 2011, 6, e23503. [CrossRef] [PubMed]

6. Aagaard, K.; Ma, J.; Antony, K.M.; Ganu, R.; Petrosino, J.; Versalovic, J. The placenta harbors a unique microbiome. Sci. Transl. Med. 2014, 6, 237ra65. [CrossRef]

7. Bearfield, C.; Davenport, E.S.; Sivapathasundaram, V.; Allaker, R.P. Possible association between amniotic fluid micro-organism infection and microflora in the mouth. BJOG 2002, 109, 527-533. [CrossRef] [PubMed]

8. Jiménez, E.; Fernández, L.; Marín, M.L.; Martín, R.; Odriozola, J.M.; Nueno-Palop, C.; Narbad, A.; Olivares, M.; Xaus, J.; Rodríguez, J.M. Isolation of commensal bacteria from umbilical cord blood of healthy neonates born by cesarean section. Curr. Microbiol. 2005, 51, 270-274. [CrossRef] [PubMed] 
9. Stout, M.J.; Conlon, B.; Landeau, M.; Lee, I.; Bower, C.; Zhao, Q.; Roehl, K.A.; Nelson, D.M.; Macones, G.A.; Mysorekar, I.U. Identification of intracellular bacteria in the basal plate of the human placenta in term and preterm gestations. Am. J. Obstet. Gynecol. 2013, 208, 226.e1-226.e7. [CrossRef] [PubMed]

10. Sampaio-Maia, B.; Monteiro-Silva, F. Acquisition and maturation of oral microbiome throughout childhood: An update. Dent. Res. J. 2014, 11, 291-301.

11. Dominguez-bello, M.G.; Costello, E.K.; Contreras, M.; Magris, M.; Hidalgo, G. Delivery mode shapes the acquisition and structure of the initial microbiota across multiple body habitats in newborns. Proc. Natl. Acad. Sci. USA 2010, 107, 11971-11975. [CrossRef] [PubMed]

12. Lif Holgerson, P.; Harnevik, L.; Hernell, O.; Tanner, A.C.; Johansson, I. Mode of birth delivery affects oral microbiota in infants. J. Dent. Res. 2011, 90, 1183-1188. [CrossRef]

13. Bäckhed, F.; Roswall, J.; Peng, Y.; Feng, Q.; Jia, H.; Kovatcheva-Datchary, P.; Li, Y.; Xia, Y.; Xie, H.; Zhong, H.; et al. Dynamics and stabilization of the human gut microbiome during the first year of life resource dynamics and stabilization of the human gut microbiome during the first year of life. Cell Host Microbe 2015, 17, 690-703. [CrossRef]

14. Filippidi, A.; Galanakis, E.; Maraki, S.; Galani, I.; Drogari-Apiranthitou, M.; Kalmanti, M.; Mantadakis, E.; Samonis, G. The effect of maternal flora on Candida colonisation in the neonate. Mycoses 2014, 57, 43-48. [CrossRef]

15. Holgerson, P.L.; Vestman, N.R.; Claesson, R.; Öhman, C.; Domellöf, M.; Tanner, A.C.; Hernell, O.; Johansson, I. Oral microbial profile discriminates breast-fed from formula-fed infants. J. Pediatr. Gastroenterol. Nutr. 2013, 56, 127-136. [CrossRef]

16. Moossavi, S.; Sepehri, S.; Robertson, B.; Bode, L.; Goruk, S.; Field, C.J.; Lix, L.M.; de Souza, R.J.; Becker, A.B.; Mandhane, P.J.; et al. Composition and variation of the human milk microbiota are influenced by maternal and early-life factors. Cell Host Microbe 2019, 25, 324-335.e4. [CrossRef]

17. Monteiro-da-Silva, F.; Araujo, R.; Sampaio-Maia, B. Interindividual variability and intraindividual stability of oral fungal microbiota over time. Med. Mycol. 2014, 52, 498-505. [CrossRef] [PubMed]

18. Monteiro-da-Silva, F.; Sampaio-Maia, B.; de Pereira, M.L.; Araujo, R. Characterization of the oral fungal microbiota in smokers and non-smokers. Eur. J. Oral Sci. 2013, 121, 132-135. [CrossRef]

19. Ghannoum, M.A.; Jurevic, R.J.; Mukherjee, P.K.; Cui, F.; Sikaroodi, M.; Naqvi, A.; Gillevet, P.M. Characterization of the oral fungal microbiome (mycobiome) in healthy individuals. Plos Pathog. 2010, 6, e1000713. [CrossRef]

20. Payne, M.S.; Cullinane, M.; Garland, S.M.; Tabrizi, S.N.; Donath, S.M.; Bennett, C.M.; Amir, L.H. Detection of Candida spp. in the vagina of a cohort of nulliparous pregnant women by culture and molecular methods: Is there an association between maternal vaginal and infant oral colonisation? Aust. N. Z. J. Obstet. Gynaecol. 2016, 56, 179-184. [CrossRef]

21. Kraneveld, E.A.; Buijs, M.J.; Bonder, M.J.; Visser, M.; Keijser, B.J.; Crielaard, W.; Zaura, E. The relation between oral candida load and bacterial microbiome profiles in Dutch older adults. PLOS ONE 2012, 7, e42770. [CrossRef]

22. Socransky, S.S.; Haffajee, A.D. Dental biofilms: Difficult therapeutic targets. Periodontol. 2000 2002, 28, 12-55. [CrossRef] [PubMed]

23. Segata, N.; Haake, S.; Mannon, P.; Lemon, K.P.; Waldron, L.; Gevers, D.; Huttenhower, C.; Izard, J. Composition of the adult digestive tract bacterial microbiome based on seven mouth surfaces, tonsils, throat and stool samples. Genome Biol. 2012, 13. [CrossRef]

24. Klimesova, K.; Jiraskova Zakostelska, Z.; Tlaskalova-Hogenova, H. Oral bacterial and fungal microbiome impacts colorectal carcinogenesis. Front. Microbiol. 2018, 9. [CrossRef]

25. Trojanowska, D.; Zwolinska-Wcislo, M.; Tokarczyk, M.; Kosowski, K.; Mach, T.; Budak, A. The role of Candida in inflammatory bowel disease. Estimation of transmission of C. albicans fungi in gastrointestinal tract based on genetic affinity between strains. Med. Sci. Monit. 2010, 16, CR451-CR457.

26. Walther-António, M.R.S.; Jeraldo, P.; Berg Miller, M.E.; Yeoman, C.J.; Nelson, K.E.; Wilson, B.A.; White, B.A.; Chia, N.; Creedon, D.J. Pregnancy's stronghold on the vaginal microbiome. PLoS ONE 2014, 9, e98514. [CrossRef]

27. Schoch, C.L.; Seifert, K.A.; Huhndorf, S.; Robert, V.; Spouge, J.L.; Levesque, C.A.; Chen, W.; Bolchacova, E.; Voigt, K.; Crous, P.W.; et al. Nuclear ribosomal internal transcribed spacer (ITS) region as a universal DNA barcode marker for Fungi. Proc. Natl. Acad. Sci. USA 2012, 109, 6241-6246. [CrossRef] 
28. Caporaso, J.G.; Kuczynski, J.; Stombaugh, J.; Bittinger, K.; Bushman, F.D.; Costello, E.K.; Fierer, N.; Peña, A.G.; Goodrich, J.K.; Gordon, J.I.; et al. QIIME allows analysis of high-throughput community sequencing data. Nat. Methods 2010, 7, 335-336. [CrossRef]

29. Edgar, R.C. Search and clustering orders of magnitude faster than BLAST. Bioinformatics 2010, 26, $2460-2461$. [CrossRef] [PubMed]

30. DeSantis, T.Z.; Hugenholtz, P.; Larsen, N.; Rojas, M.; Brodie, E.L.; Keller, K.; Huber, T.; Dalevi, D.; Hu, P.; Andersen, G.L. Greengenes, a Chimera-Checked 16S rRNA gene database and workbench compatible with ARB. Appl. Environ. Microbiol. 2006, 72, 5069-5072. [CrossRef]

31. Lozupone, C.; Knight, R. UniFrac: A new phylogenetic method for comparing microbial communities. Appl. Environ. Microbiol. 2005, 71, 8228-8235. [CrossRef]

32. Bender, R.; Lange, S. Adjusting for multiple testing-when and how? J. Clin. Epidemiol. 2001, 54, 343-349. [CrossRef]

33. Al-Shehri, S.S.; Sweeney, E.L.; Cowley, D.M.; Liley, H.G.; Ranasinghe, P.D.; Charles, B.G.; Shaw, P.N.; Vagenas, D.; Duley, J.A.; Knox, C.L. Deep sequencing of the $16 \mathrm{~S}$ ribosomal RNA of the neonatal oral microbiome: A comparison of breast-fed and formula-fed infants. Sci. Rep. 2016, 6, 38309. [CrossRef]

34. Costello, E.K.; Carlisle, E.M.; Bik, E.M.; Morowitz, M.J.; Relman, D.A. Microbiome assembly across multiple body sites in low-birthweight infants. mBio 2013, 4. [CrossRef]

35. Ling, Z.; Kong, J.; Jia, P.; Wei, C.; Wang, Y.; Pan, Z.; Huang, W.; Li, L.; Chen, H.; Xiang, C. Analysis of oral microbiota in children with dental caries by PCR-DGGE and barcoded pyrosequencing. Microb. Ecol. 2010, 60, 677-690. [CrossRef]

36. Lazarevic, V.; Whiteson, K.; Huse, S.; Hernandez, D.; Farinelli, L.; Østerås, M.; Schrenzel, J.; François, P. Metagenomic study of the oral microbiota by Illumina high-throughput sequencing. J. Microbiol. Methods 2009, 79, 266-271. [CrossRef]

37. Keijser, B.J.F.; Zaura, E.; Huse, S.M.; van der Vossen, J.M.B.M.; Schuren, F.H.J.; Montijn, R.C.; ten Cate, J.M.; Crielaard, W. Pyrosequencing analysis of the oral microflora of healthy adults. J. Dent. Res. 2008, 87, 1016-1020. [CrossRef]

38. Könönen, E.; Jousimies-Somer, H.; Bryk, A.; Kilpi, T.; Kilian, M. Establishment of streptococci in the upper respiratory tract: Longitudinal changes in the mouth and nasopharynx up to 2 years of age. J. Med. Microbiol. 2002, 51, 723-730. [CrossRef]

39. Könönen, E.; Asikainen, S.; Jousimies-Somer, H. The early colonization of gram-negative anaerobic bacteria in edentulous infants. Oral Microbiol. Immunol. 1992, 7, 28-31. [CrossRef]

40. Könönen, E.; Asikainen, S.; Saarela, M.; Karjalainen, J.; Jousimies-Somer, H. The oral gram-negative anaerobic microflora in young children: Longitudinal changes from edentulous to dentate mouth. Oral Microbiol. Immunol. 1994, 9, 136-141. [CrossRef] [PubMed]

41. Kolenbrander, P.E.; Palmer, R.J.; Periasamy, S.; Jakubovics, N.S. Oral multispecies biofilm development and the key role of cell-cell distance. Nat. Rev. Microbiol. 2010, 8, 471-480. [CrossRef]

42. Collado, M.C.; Delgado, S.; Maldonado, A.; Rodríguez, J.M. Assessment of the bacterial diversity of breast milk of healthy women by quantitative real-time PCR. Lett. Appl. Microbiol. 2009, 48, 523-528. [CrossRef]

43. Aas, J.A.; Griffen, A.L.; Dardis, S.R.; Lee, A.M.; Olsen, I.; Dewhirst, F.E.; Leys, E.J.; Paster, B.J. Bacteria of dental caries in primary and permanent teeth in children and young adults. J. Clin. Microbiol. 2008, 46, 1407-1417. [CrossRef]

44. Mantzourani, M.; Gilbert, S.C.; Sulong, H.N.H.; Sheehy, E.C.; Tank, S.; Fenlon, M.; Beighton, D. The isolation of bifidobacteria from occlusal carious lesions in children and adults. Caries Res. 2009, 43, 308-313. [CrossRef]

45. Kanasi, E.; Dewhirst, F.E.; Chalmers, N.I.; Kent, R., Jr.; Moore, A.; Hughes, C.V.; Pradhan, N.; Loo, C.Y.; Tanner, A.C. Clonal analysis of the microbiota of severe early childhood caries. Caries Res. 2010, 44, 485-497. [CrossRef]

46. Tanner, A.C.R.; Mathney, J.M.J.; Kent, R.L.; Chalmers, N.I.; Hughes, C.V.; Loo, C.Y.; Pradhan, N.; Kanasi, E.; Hwang, J.; Dahlan, M.A.; et al. Cultivable Anaerobic microbiota of severe early childhood caries. J. Clin. Microbiol. 2011, 49, 1464-1474. [CrossRef]

47. Tham, R.; Bowatte, G.; Dharmage, S.C.; Tan, D.J.; Lau, M.X.; Dai, X.; Allen, K.J.; Lodge, C.J. Breastfeeding and the risk of dental caries: A systematic review and meta-analysis. Acta Paediatr. 2015, 104, 62-84. [CrossRef]

48. Hegde, S.; Munshi, A.K. Influence of the maternal vaginal microbiota on the oral microbiota of the newborn. J. Clin. Pediatr. Dent. 1998, 22, 317-321.

49. Bagg, J.; MacFarlane, T.W.; Poxton, I.R.; Smith, A.J. Essentials of Microbiology for Dental Students; Oxford University Press: New York, NY, USA, 2006; ISBN 0198564899. 
50. Peters, B.A.; Wu, J.; Hayes, R.B.; Ahn, J. The oral fungal mycobiome: Characteristics and relation to periodontitis in a pilot study. BMC Microbiol. 2017, 17. [CrossRef]

51. Dinleyici, M.; Pérez-Brocal, V.; Arslanoglu, S.; Aydemir, O.; Ozumut, S.S.; Tekin, N.; Vandenplas, Y.; Moya, A.; Dinleyici, E.C. Human milk mycobiota composition: Relationship with gestational age, delivery mode, and birth weight. Benef. Microbes 2020, 11, 151-162. [CrossRef]

Publisher's Note: MDPI stays neutral with regard to jurisdictional claims in published maps and institutional affiliations.

(C) 2020 by the authors. Licensee MDPI, Basel, Switzerland. This article is an open access article distributed under the terms and conditions of the Creative Commons Attribution (CC BY) license (http://creativecommons.org/licenses/by/4.0/). 